\title{
Dental prophylaxis influence in tooth color assessment-Clinical study
}

\author{
Ruben Pereira DDS, MSc ${ }^{1}$ ～Daniela Corado DDS, $\mathrm{MSc}^{1}$ ～João Silveira DDS, $\mathrm{PhD}^{2}$ | \\ Rita Alves DDS, MSc ${ }^{1}$ | António Mata DDS, PhD, FICD ${ }^{2}$ | Duarte Marques DDS, $\mathrm{PhD}^{2}$
}

${ }^{1}$ Oral Biology and Biochemistry Research Group - Faculty of Dental Medicine, Universidade de Lisboa, Lisbon, Portugal

${ }^{2}$ Oral Biology and Biochemistry Research Group - LIB-Phys FCT UID/FIS/04559/2013, Faculty of Dental Medicine, Universidade de Lisboa, Lisbon, Portugal

\section{Correspondence}

Duarte Marques, Oral Biology and Biochemistry Research Group - LIB-Phys, Faculty of Dental Medicine, Universidade de Lisboa, Rua Professora Teresa Ambrósio, Cidade Universitária, 1600-277 Lisbon, Portugal.

Email: duarte.marques@campus.ul.pt

\begin{abstract}
Objective: To evaluate dental prophylaxis influence in tooth color assessment with the use of different methods.

Materials and methods: Volunteers were consecutively recruited and screened according to appropriate inclusion/exclusion criteria. Each participant's upper right central incisive and canine color was measured before and after dental prophylaxis with a one-week interval. Tooth color assessment was performed by calibrated operators and the patient using a VITA Classical shade guide and by spectrophotometric methods with a proper device (SpectroShade). Color was reported as mean with SD of Commission Internationale De l'Eclairage (CIE) $L^{*} a^{*} b^{*}$ values, $\Delta E_{\mathrm{ab}}$ and $\Delta E_{00}$. Oneway analysis of variance and Tukey post hoc were performed to assess differences between methods and paired $t$ test for assessing differences in tooth color coordinates after dental prophylaxis. Values of $P<.05$ were taken as significant.

Results: Fifty patients were included. Self-assessed dental prophylaxis effects equated to a significantly different mean $\Delta E_{00}$ value of $2.3 \pm 1.7(P<.01)$, when compared to the calibrated operator reported $0.8 \pm 1.1$ and the spectrophotometer $1.0 \pm 0.5$. Half of volunteer's $\Delta E_{00}$ values surpassed the acceptability threshold, when compared to $28 \%$ from investigator and $10 \%$ from spectrophotometer.

Conclusion: Performing a dental prophylaxis did have an influence in tooth color perception with a higher self-perceived effect in the patient assessment.

Clinical significance: The results of this study suggest that performing professional dental prophylaxis presented a perceived effect in tooth color regardless of the method used being that patients precepted whiter and less yellowish teeth. Since extrinsic stain is considered as one of the factors that could influence tooth color assessment, performing professional dental prophylaxis prior to composite or ceramic color selection in anterior teeth restorations could consequently increase treatment predictability.
\end{abstract}

\section{KEYWORDS}

dental prophylaxis, tooth, color, aesthetics, spectrophotometry 


\section{1 | INTRODUCTION}

Nowadays a harmonious and beautiful smile is considered a major aesthetic attribute with patient's aesthetic demands increasing over the last years. ${ }^{1,2}$ Among the main factors that influence smile perception, such as tooth dimension and shape, gingival anatomy or lips position, tooth color is considered as one of the most important. ${ }^{3}$ The demand for tooth bleaching treatments is increasing in the daily clinical practice since lighter and whiter teeth are considered as representative of an aesthetic smile. ${ }^{1,2,4}$

Tooth color assessment is a complex procedure in which several factors can influence the results, such as light conditions, background color, and observer-related factors (eg, experience in color assessment, eye fatigue, emotional state, age, genre).$^{5-7}$ This is a subjective procedure with visual assessment differing from different observers. ${ }^{8}$ Additionally, tooth color self-perception is also inconstant and has an important role in aesthetic treatments results being associated with patients' satisfaction. ${ }^{9}$ Because of tooth color assessment subjectivity, dental manufacturer's, in the last years, aimed to develop new equipment that could objectively assess tooth color such as light standardization devices, dental spectrophotometers and colorimeters or imaging systems. ${ }^{10-13}$

Tooth extrinsic staining occurs daily and, without regular ora hygiene appointments, their increase may interfere in tooth color assessment, which could be relevant for the patient self-perception or even interfere with the results of a bleaching treatment. ${ }^{14-17}$

The present clinical study aimed to evaluate the influence of dental prophylaxis in tooth color assessment in healthy patients. For that purpose, the following null hypothesis was established: there was no difference in tooth color assessment before and after a dental prophylaxis.

As secondary objectives for the study, we intended to assess agreements between different tooth color methods with different light conditions.

\section{2 | MATERIALS AND METHODS}

This clinical diagnostic study was conducted in full compliance with the Helsinki World Medical Association Declaration and its most recent amendments, being approved by the local ethics committee and registered at the US National Library of Medicine ClinicalTrials. gov website under the reference number NCT03544658.

\section{1 | Sample size calculation}

A power analysis to obtain an adequate sample size was previously performed with the data from our pilot study using an online calculator (www.sealedenvelope.com). ${ }^{18}$ Considering the mean of Delta $E$ $\left(\Delta E_{\mathrm{ab}}\right)=1.6$ in the spectrophotometer, we established that at least 40 patients would be needed for an equivalence limit equal to the value of 1.2 units of $\Delta E$ (the value for the perceptibility threshold [PT]) with a power of $80 \%$ and $\alpha$ of $5 \%$. To offset a possible attrition bias, $25 \%$ was added to the total sample, resulting in a total of 50 participants.

\section{2 | Participants}

A total of 62 volunteers who attended the Faculty of Dentistry clinic of the Universidade de Lisboa, were screened according to the following inclusion criteria: to be at least 18 years old and at least one of the selected teeth (upper right central incisive-11; upper right canine13) darker than A3 in VITA Classical shade guide (assessed by the spectrophotometer). Exclusion criteria were the presence of fixed orthodontic appliances, pregnancy, smokers, color vision deficiency, previous professional dental prophylaxis performed in a period shorter than 6 months before the first appointment, upper central incisors and upper canines with dental restorations, endodontic treatment or decay in anterior teeth and severe anomalies of the dental structure. Study design is summarized in Figure 1.

\subsection{Intervention and measures}

In the first appointment, tooth color for the 11 and 13 teeth of the participants were visually assessed using VITA Classical shade guide (VC) (VITA Zahnfabrick, Germany; B027C and B027CV1), organized by value and converted to $\mathrm{CIE} L^{*} a^{*} b^{*}$ system according to O'Brien et al. ${ }^{19}$ Tooth color measurements were performed by the volunteer and a calibrated investigator with the patient seated in high Fowler's position on the dental chair with daylight conditions. To standardize these conditions, measurements were always performed in the same dental office with daylight source from an open window, at the same day hour (approximately 10 AM-12 PM). A second visual shade assessment was performed using a standard light device, Smile Lite (SL) (Smile Line AS, Switzerland; serial number 052015), with LED lights at $5500 \mathrm{~K}$ and a polarization filter, according to manufacturer's instructions. The calibrated investigator was a dentist with a minimum of 3 years' clinical experience, a negative history of visual color deficiencies (confirmed using X-Rite Color Challenge by Pantone) and submitted to a calibration process. This process was conducted by consecutive determination of VC visual shade guides using two VC scales (one of them with blinded shade guide identification) and the dentist would be considered a valid operator if obtained an intraclass correlation coefficient (ICC) higher than 0.80 (considered as excellent reliability ${ }^{20}$ ). Obtained ICC for the total of three calibrated operators ranged between 0.85 and 0.91 .

The participants performed shade matching with an extraoral mirror in front of them held by a third independent operator which registered the measurement and did not take part in visual color assessment procedures, being that participant and investigator were blind to the measurements performed in the other groups.

A spectrophotometer, SpectroShade micro (SS) (MHT Optic Research, Niederhasli, Switzerland; serial number HDL3973), was used as a gold standard measurement method, since it is considered 
FIGURE 1 Study design

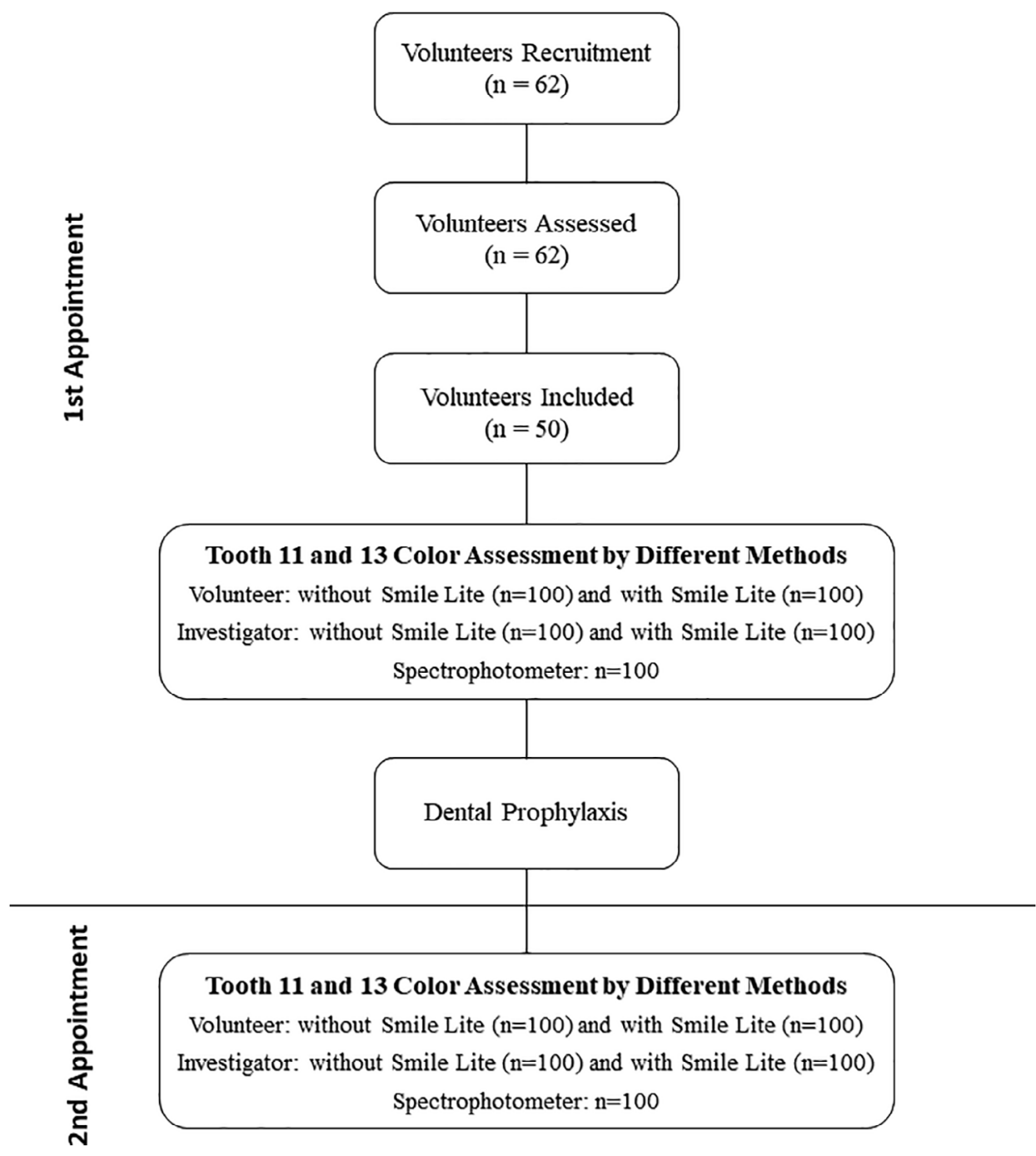

as a diagnostic test device with high levels of reproducibility, requiring low level of training and with good matching with the standard tooth shade guides. ${ }^{5,11,21-24}$ The SS was operated by an independent investigator, according to manufacturer's instructions, performing three measuring rounds. Results were registered in VC shade guide and $\mathrm{CIE}$ $L^{*} a^{*} b^{*}$ system. ${ }^{25-27}$

Following tooth color assessment, every patient was submitted to a professional dental prophylaxis using an ultrasonic scaler and a nylon brush with prophylaxis paste (Cleanic, Kerr Orange) in low rotation contra-angle handpiece by a dentist. After 1 week, tooth color was assessed as previously described for the first appointment.

\subsection{Statistical analysis}

All data collected were analyzed using IBM SPSS version 24 (IBM Statistics, Inc., Chicago, Illinois). Parametric tests were used since the study had a sufficiently large sample size according to the central limit theorem. ${ }^{28}$ The kappa factor and asymptotic error were determined to assess the agreement between tooth color assessment methods in the different appointments and classified as less than 0.20-poor agreement, 0.21 to $0.4-$ fair agreement, 0.41 to 0.6 moderate agreement, 0.61 to 0.8 -good agreement, 0.81 to 1.00 -excellent agreement by previously established methods. ${ }^{29}$

Mean and SD in color change CIE $L^{*} a^{*} b^{*}$ parameters were determined and used to calculate $\Delta E$ value representing the color difference after the dental prophylaxis effect. Student's paired $t$ tests were conducted to analyze intragroup differences in $\mathrm{CIE} L^{*} a^{*} b^{*}$ values and one-way analysis of variance with Tukey post hoc were used to determine differences in $\Delta E$ values between tooth color assessment group methods.

To calculate color coordinates differences, it was used formulas of the CIE: the CIELAB formula $\left(\Delta E_{\mathrm{ab}}\right)$ and the CIEDE2000 formula $\Delta E_{00}$. Computations with these color difference formulas were performed according to the following equations $^{30}: \Delta E_{a b}=\sqrt{\left(L_{2}-L_{1}\right)^{2}+\left(a_{2}-a_{1}\right)^{2}\left(b_{2}-b_{1}\right)^{2}}$

$$
\Delta E_{00}=\sqrt{\left(\frac{L_{2}-L_{1}}{K_{L} S_{L}}\right)^{2}+\left(\frac{C_{2}-C_{1}}{K_{C} S_{C}}\right)^{2}+\left(\frac{H_{2}-H_{1}}{K_{H} S_{H}}\right)^{2}+R_{T}\left(\frac{C_{2}-C_{1}}{K_{C} S_{C}}\right)\left(\frac{H_{2}-H_{1}}{K_{H} S_{H}}\right)}
$$

All parametric factors were set to 1 . Color difference perception was assessed with two major thresholds: PT considered as 
$\Delta E_{\mathrm{ab}}=1.2 / \Delta E_{00}=0.8$ and acceptability threshold (AT) considered as $\Delta E_{\mathrm{ab}}=2.7 / \Delta E_{\mathrm{OO}}=1.8 .^{31-33}$

\section{3 | RESULTS}

Fifty participants were included in the study after the recruitment procedures, 36 females and 14 males with ages ranging between 18 and 43 years old. A total of 100 teeth were evaluated by the participants, calibrated operators and spectrophotometer without dropouts in between appointments.

Table 1 summarizes the descriptive analysis of $L^{*} a^{*} b^{*}$ mean values and SD obtained in the different group methods, before and after professional dental prophylaxis, without statistical differences between groups or coordinates. However, self-perception, with a mean $\Delta E_{\mathrm{ab}}=3.2 \pm 2.4$ and mean $\Delta E_{00}=2.3 \pm 1.7$ presented the highest color difference (Table 2 ), with statistically significant differences when compared to the calibrated clinician and the SS mean

TAB LE 1 CIE $L^{*} a^{*} b^{*}$ values expressed as mean and SD before (first appointment) and after (second appointment) dental prophylaxis

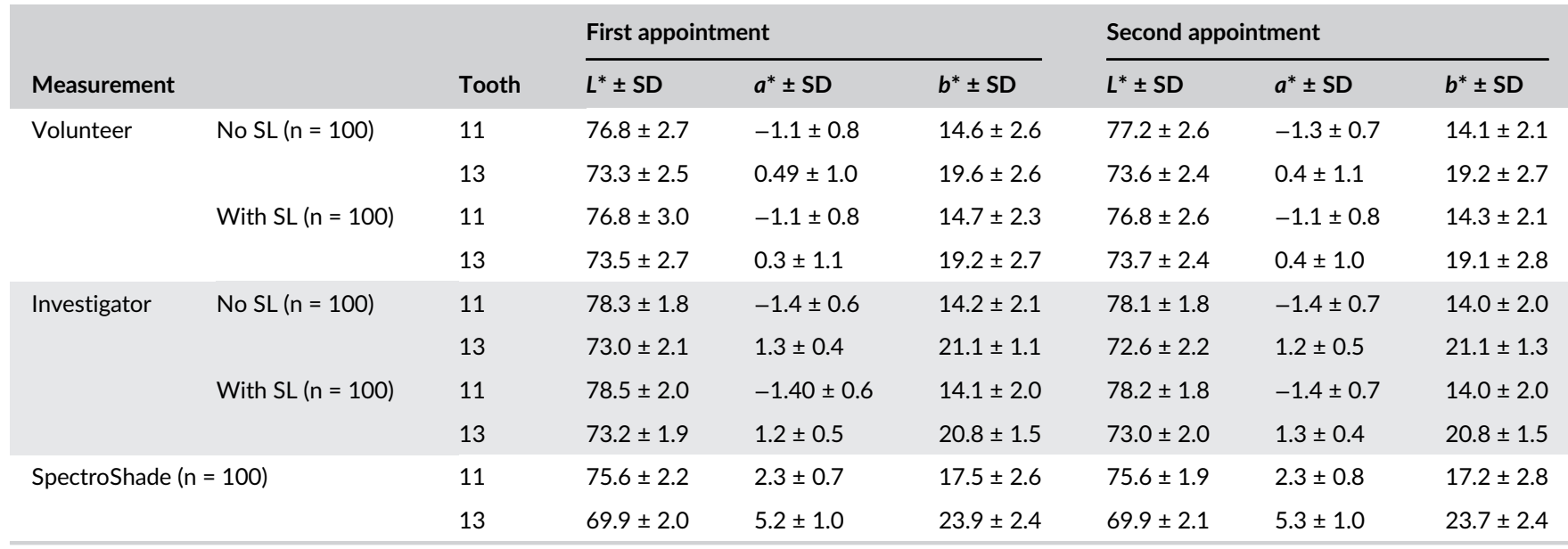

Abbreviation: SD, standard deviation; SL, Smile Lite.

TABLE $2 \Delta E_{\mathrm{ab}}$ and $\Delta E_{00}$ mean value and SD after dental prophylaxis. Also presented the number of cases where $\Delta E_{\mathrm{ab}}$ and $\Delta E_{00}$ were higher than the perceptibility $\left(\Delta E_{\mathrm{ab}}=1.2 ; \Delta E_{00}=0.8\right)$ and acceptability $\left(\Delta E_{\mathrm{ab}}=2.7 ; \Delta E_{00}=1.8\right)$ thresholds and $95 \% \mathrm{Cl}$

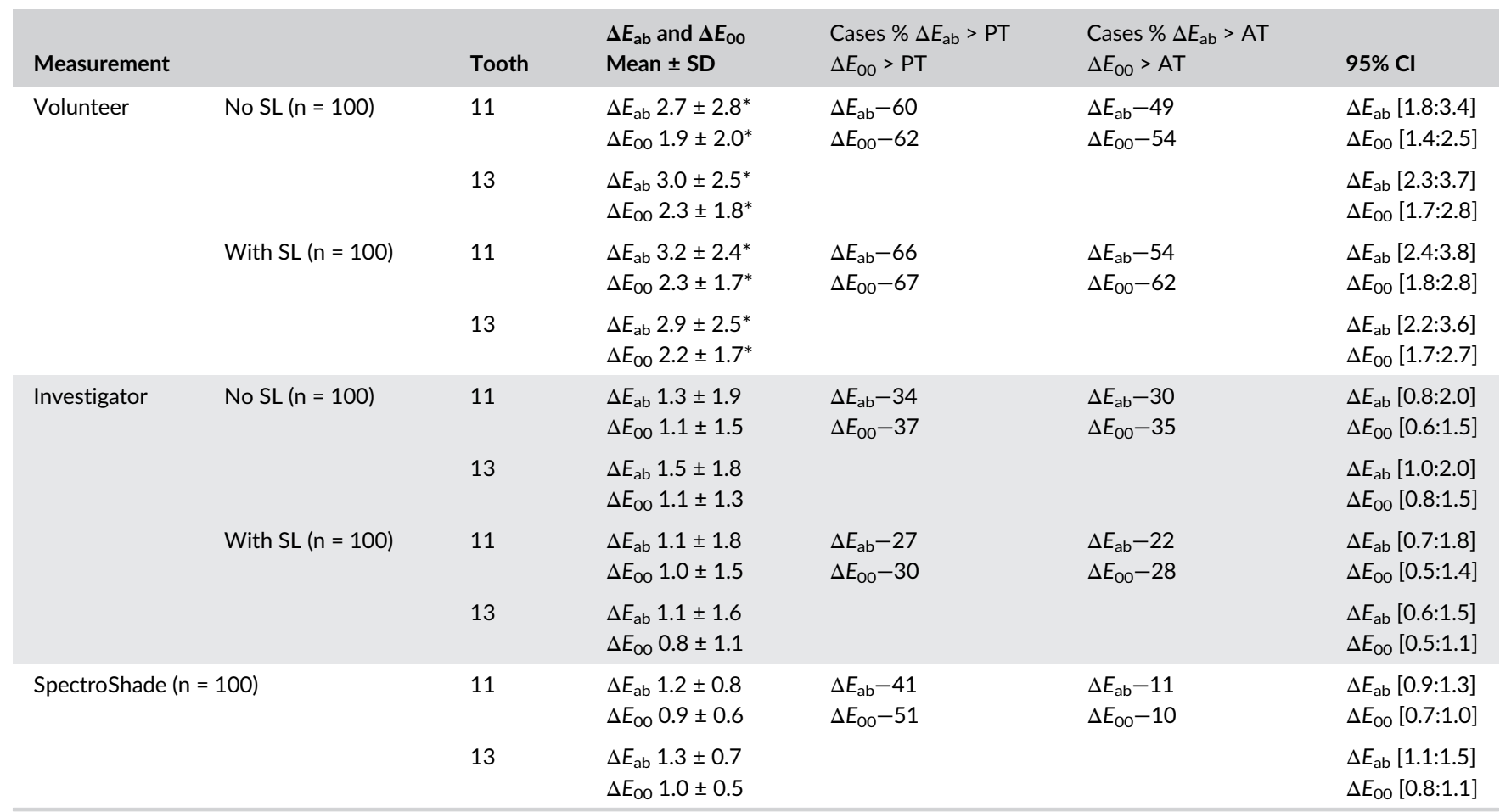

Note: Asterisks represent statistically significant differences-volunteer $\Delta E_{00}$ and $\Delta E_{\mathrm{ab}}$ values were different from Investigator and SpectroShade $(P<.05)$. Abbreviations: AT, acceptability threshold; $\mathrm{Cl}$, confidence interval; PT, perceptibility threshold; SD, standard deviation; SL, Smile Lite. 
TA B LE 3 Kappa agreement and asymptotic error related to methods and light conditions for tooth color measurements in both study's appointments

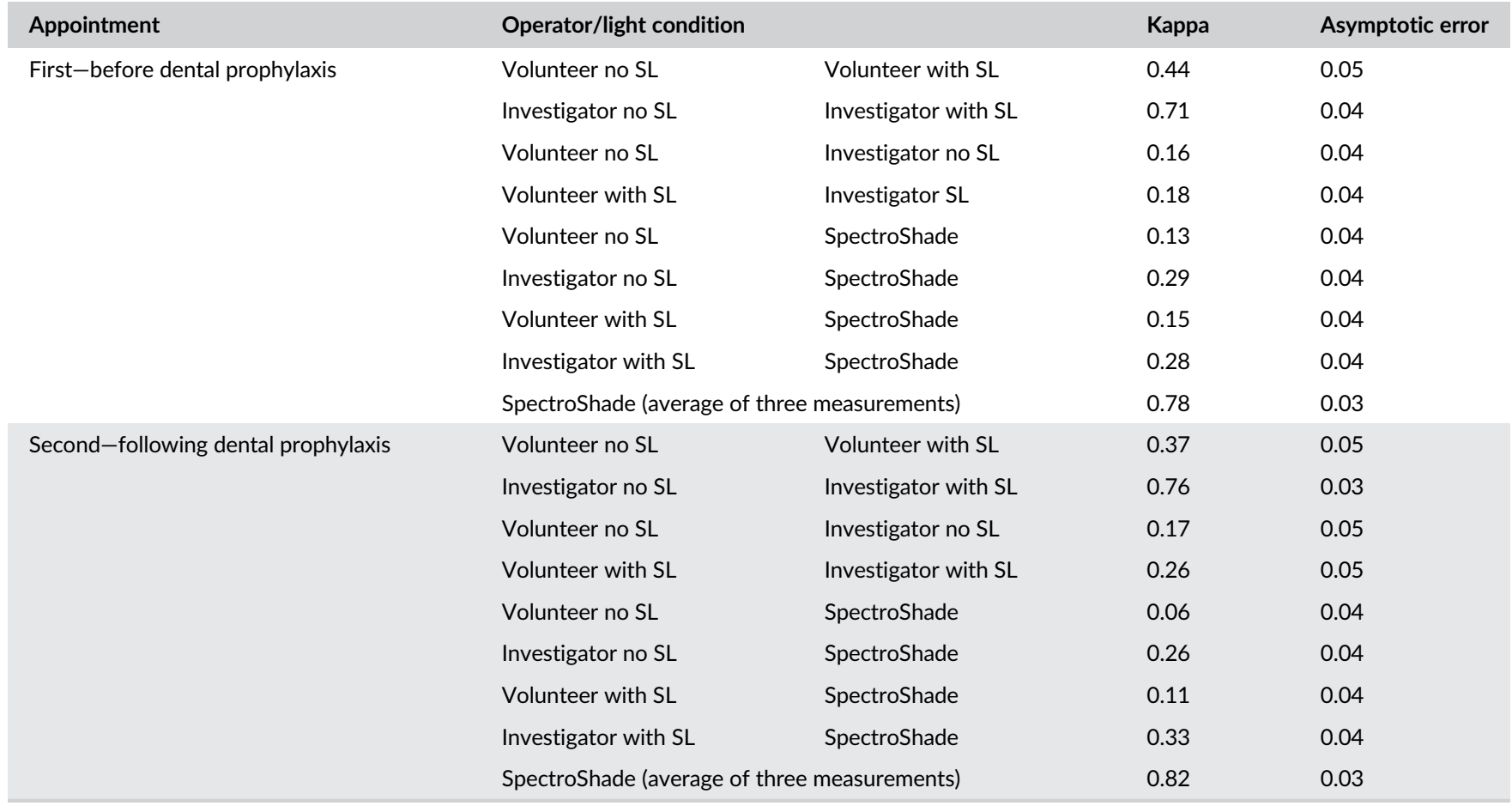

Abbreviation: SL, Smile Lite.

values (Table 2). Additionally, when assessing the percentage of teeth that the $\Delta E_{\mathrm{ab}} / \Delta E_{00}$ values surpassed the $\mathrm{AT}$, results equated to $51.5 \% / 58.0 \%, 26.0 \% / 31.5 \%$, and $11.0 \% / 10.0 \%$ for the volunteer, calibrated clinician, and SS groups, respectively. Similar results occurred when tooth color assessment was performed with SL, despite the decrease in $\Delta E_{\mathrm{ab}} / \Delta E_{00}$ mean (Table 2).

Table 3 summarizes the agreement, before and after dental prophylaxis, in the different group methods and the SS internal agreement. In five out of nine comparisons, kappa factor coefficient was better after extrinsic stain removal. The results presented a higher agreement between the investigator and the SS ( $k=0.26$, fair agreement) when compared with the volunteer ( $k=0.06$, poor agreement). In almost all cases (83.3\%), the use of SL increased the agreement between visual tooth color assessment methods and the SS.

\section{4 | DISCUSSION}

This clinical diagnostic study evaluated the influence of dental prophylaxis in tooth color assessment of healthy patients. Our results suggest that dental prophylaxis influences tooth color assessment with a higher effect in tooth color self-perception when compared to the other used methods, with $50 \%$ of patients detecting whiter teeth above the AT after the intervention, thus rejecting the null hypothesis.

This study was designed to assess the influence of clinical experience, presence of extrinsic pigmentation and use of a spectrophotometer in tooth color assessment regarding intramethod and intermethod concordances.
After dental prophylaxis, patients presented a different tooth color perception when compared with experienced operators whose results were similar to the spectrophotometer. Although experience could be an influencing factor in tooth color assessment, it is controversially presented in literature. In studies conducted by Gasparik et al, ${ }^{10,34}$ inexperienced students reported no differences in tooth color assessment when compared to trained dentists in different light situations, which are in agreement with results from other studies. ${ }^{35,36}$ However, in a study conducted by Della Bona et al, ${ }^{8}$ assessing the agreement between visual and instrumental selection, it was found that experienced dentists presented a higher agreement compared with nonexperienced subjects, regardless of shade guides and lighting conditions. Furthermore, some studies concluded that training dental students with computer software significantly improved shade matching. ${ }^{37,38}$ The study conducted by Corcodel et al ${ }^{39}$ concluded that the use of a group-learning approach in a clinical setting could improve the shade-matching performance ability in dental students.

Evaluating the effect of experience or visual procedure when compared to the spectrophotometric assessment presented an operator fair agreement ( $k=0.29$ with a $4 \%$ error) which is far from the ideal value of $k=0.80$ but higher than the volunteer group which presented poor agreement ( $k=0.15$ with a $4 \%$ error). Therefore, and comparing with the results mentioned in the literature, the use of a spectrophotometer continues to be the method with higher agreement. ${ }^{5,11,21-24}$ However, in our study the intradevice agreement increased after performing dental prophylaxis which suggests that extrinsic stains could be a possible confounding factor in tooth color 
determination, and therefore should be removed previous to aesthetical treatments to increase clinical predictability. These findings were also reported in literature, ${ }^{14-17}$ although only from in vitro studies thus requiring clinical validation.

Some studies ${ }^{10,26,34}$ reported that light conditions may influence tooth color assessment, which could be controlled if a standard environment is preestablished. For that reason, in this study was decided to assess if the use of a light standardization device could improve the agreement between different methods. Although an agreement increase was observed in $83.3 \%$ comparisons, optimal kappa factor coefficient levels were never obtained $(k>0.80)$ which could be due to other reported tooth color assessment confounding factors. ${ }^{6,7,14}$

Our study sample was constituted mainly by young individuals who have an overall good oral hygiene, with regular 6-month to 1-year dental appointments which could lead to lower extrinsic pigmentation. Even so, we detected a dental prophylaxis effect which leads us to assume that in older patients, who do not attend dental appointments regularly, a greater effect could be detected.

The appearance and tooth color are a common concern for patients worldwide and is associated with an increased desire for treatments that improve dental aesthetics. Extrinsic stain as confounding factor for tooth color determination makes professional dental prophylaxis an advisable procedure before aesthetic treatments. Patient self-perception may benefit from it and higher satisfaction levels could be achieved. In the future, further investigations in older populations, with higher levels of extrinsic stains (eg, smokers) or with different dental appointment routines may be pertinent.

\section{5 | CONCLUSIONS}

Performing a professional dental prophylaxis evoked an influence in tooth color assessment with a higher effect in the patient's tooth color self-perception.

\section{ACKNOWLEDGMENTS}

The authors thank the members of Oral Biology and Biochemistry Research Group for their support and valuable help in conducting this research and FMDUL for providing the facilities to conduct the study.

\section{CONFLICT OF INTEREST}

The authors do not have any financial interest in the companies whose materials are included in this article.

\section{ORCID}

Duarte Marques (D) https://orcid.org/0000-0003-1966-8281

\section{REFERENCES}

1. Majeed A, Farooq I, Grobler SR, Rossouw RJ. Tooth-bleaching: a review of the efficacy and adverse effects of various tooth whitening products. J Coll Physicians Surg Pak. 2015;25:891-896.

2. Araujo E, Ballarin A, Briso A, et al. Tooth Whitening: An Evidence-Based Perspective. Minneapolis, MN: Springer; 2016.
3. Machado A. 10 commandments of smile esthetics. Dental Press J Orthod. 2014;19:136-157.

4. Demarco FF, Meireles SS, Masotti AS. Over-the-counter whitening agents: a concise review. Braz Oral Res. 2009;1:64-70.

5. Ishikawa-Nagai S, Yoshida A, Da Silva JD, Miller L. Spectrophotometric analysis of tooth color reproduction on anterior all-ceramic crowns: part 1: analysis and interpretation of tooth color. J Esthet Restor Dent. 2010;22:42-52.

6. Joiner A, Hopkinson I, Deng Y, Westland S. A review of tooth colour and whiteness. J Dent. 2008;1:S2-S7.

7. Joiner A, Luo W. Tooth colour and whiteness: a review. J Dent. 2017; 67S:S3-S10.

8. Della Bona A, Barrett AA, Rosa V, Pinzetta C. Visual and instrumental agreement in dental shade selection: three distinct observer populations and shade matching protocols. Dent Mater. 2009;25:276-281.

9. Tin-Oo MM, Saddki N, Hassan N. Factors influencing patient satisfaction with dental appearance and treatments they desire to improve aesthetics. BMC Oral Health. 2011;11:6.

10. Gasparik C, Tofan A, Culic B, Badea M, Dudea D. Influence of light source and clinical experience on shade matching. Clujul Med. 2014;87:30-33.

11. Chu SJ, Trushkowsky RD, Paravina RD. Dental color matching instruments and systems. Review of clinical and research aspects. J Dent. 2010;38:e2-e16.

12. Klotz AL, Habibi Y, Corcodel N, Rammelsberg P, Hassel AJ, Zenthofer A. Laboratory and clinical reliability of two spectrophotometers. J Esthet Restor Dent. 2018;1-5.

13. Miyajiwala JS, Kheur MG, Patankar AH, Lakha TA. Comparison of photographic and conventional methods for tooth shade selection: a clinical evaluation. J Indian Prosthodont Soc. 2017;3:273-281.

14. Joiner A. Tooth colour: a review of the literature. J Dent. 2004;1:3-12.

15. Brook AH, Smith RN, Lath DJ. The clinical measurement of tooth colour and stain. Int Dent J. 2007;57:324-330.

16. Wang C, Lucas R, Smith AJ, Cooper PR. An in vitro screening assay for dental stain cleaning. BMC Oral Health. 2017;17:37.

17. de Geus JL, de Lara MB, Hanzen TA, et al. One-year follow-up of athome bleaching in smokers before and after dental prophylaxis. $J$ Dent. 2015;43:1346-1351.

18. Corado D. Efeito da Pigmentação Extrínseca na Perceção da Cor Dentária-Estudo In Vivo. Lisbon, Portugal: Faculdade de Medicina Dentária da Universidade de Lisboa; 2017.

19. O'Brien WJ, Groh CL, Boenke KM. A new, small-color-difference equation for dental shades. J Dent Res. 1990;69:1762-1764.

20. Fleiss J. The Design and Analysis of Clinical Experiments. New York, NY: John Wiley \& Sons, Inc.; 1986.

21. Dias S, Marques D, Silveira J, Corado D, Alves R, Mata A. Determinação da cor de diferentes escalas por dois métodos espectrofotométricos-estudo in vitro. Rev Port Estomatol Med Dent Cir Maxilofac. 2017;58:168-174.

22. Yoshida A, Miller L, Da Silva JD, Ishikawa-Nagai S. Spectrophotometric analysis of tooth color reproduction on anterior all-ceramic crowns: part 2: color reproduction and its transfer from in vitro to in vivo. J Esthet Restor Dent. 2010;22:53-63.

23. Kim-Pusateri S, Brewer JD, Davis EL, Wee AG. Reliability and accuracy of four dental shade-matching devices. J Prosthet Dent. 2009; 101:193-199.

24. Khurana R, Tredwin CJ, Weisbloom M, Moles DR. A clinical evaluation of the individual repeatability of three commercially available colour measuring devices. BDJ. 2007;203:675-680.

25. Seghi RR, Johnston WM, O'Brien WJ. Spectrophotometric analysis of color differences between porcelain systems. J Prosthet Dent. 1986:56:35-40.

26. Park JH, Lee YK, Lim BS. Influence of illuminants on the color distribution of shade guides. J Prosthet Dent. 2006;96:402-411.

27. Paravina RD, Johnston WM, Powers JM. New shade guide for evaluation of tooth whitening-colorimetric study. J Esthet Restor Dent. 2007;19:276-283. 
28. Hogg RV, Tanis EA, Zimmerman DL. Probability and Statistical Inference. New Jersey: Pearson Education; 2015:548.

29. Flight L, Julious SA. The disagreeable behaviour of the kappa statistic. Pharm Stat. 2015;14:74-78.

30. Commision Internationale de L'Eclairage. CIE 15:2004 Technical Report. Colorimetry. 3rd ed, Vienna, Austria. 2004.

31. International Organization for Standardization. ISO/TR 28642 Dentistry-Guidance on Color Measurement. Geneva: International Organization for Standardization; 2016.

32. Paravina RD, Ghinea R, Herrera LJ, et al. Color difference thresholds in dentistry. J Esthet Restor Dent. 2015;27:S1-S9.

33. Paravina RD, Perez MM, Ghinea R. Acceptability and perceptibility thresholds in dentistry: a comprehensive review of clinical and research applications. J Esthet Restor Dent. 2019;31(2):103-112.

34. Gasparik C, Grecu AG, Culic B, Badea ME, Dudea D. Shade-matching performance using a new light-correcting device. J Esthet Restor Dent. 2015;27:285-292.

35. Curd FM, Jasinevicius TR, Graves A, Cox V, Sadan A. Comparison of the shade matching ability of dental students using two light sources. J Prosthet Dent. 2006;96:391-396.
36. Bahannan SA. Shade matching quality among dental students using visual and instrumental methods. J Dent. 2014;42:48-52.

37. Borbely J, Varsanyi B, Fejerdy P, Hermann P, Jakstat HA. Toothguide trainer tests with color vision deficiency simulation monitor. J Dent. 2010;38:1-9.

38. Olms C, Klinke T, Pirek P, Hannak WB. Randomized multi-centre study on the effect of training on tooth shade matching. J Dent. 2013;41:1259-1263.

39. Corcodel N, Karatzogiannis E, Rammelsberg P, Hassel AJ. Evaluation of two different approaches to learning shade matching in dentistry. Acta Odontol Scand. 2012;70:83-88.

How to cite this article: Pereira R, Corado D, Silveira J,

Alves R, Mata A, Marques D. Dental prophylaxis influence in tooth color assessment-Clinical study. J Esthet Restor Dent.

2020;1-7. https://doi.org/10.1111/jerd.12593 